\title{
COVERINGS AND BETTI NUMBERS
}

\section{BENO ECKMANN}

1. Introduction. Let the finite polyhedron $P$ be a regular covering of the polyhedron $\bar{P}$, and let $G$ be the corresponding (finite) group of covering transformations of $P$. Then $G$ acts as operator group on the homology groups $H_{n}$ of $P$. If we consider homology with real coefficients, $H_{n}$ is a real vector group of finite rank $p_{n}=n$th Betti number of $P$, and $G$ operates in $H_{n}$ as a group of linear transformations; we denote by $s_{n}(x), x \in G$, the character of this linear representation of $G$ of degree $p_{n}$. Let $g$ be the order of $G$.

In this note we shall prove:

TheOREM 1. The nth Betti number $\bar{p}_{n}$ of $\bar{P}$ is given by

$$
p_{n}=\frac{1}{g} \sum_{x \in G} s_{n}(x) .
$$

In the Princeton Bicentennial Conference W. Hurewicz raised the question whether the homology groups of a polyhedron $\bar{P}$ are determined by those of a regular covering $P$ given as groups with operators. According to Theorem 1 the answer is affirmative in the case of finite polyhedra $P, \bar{P}$ and real (or rational) coefficients. We shall show elsewhere-in a general theory of complexes with automorphisms - that the same is true for arbitrary (finite or infinite) polyhedra, provided that the group $G$ is finite. Since the proof in our present case, based upon "harmonic chains," is very simple and yields the explicit formula of Theorem 1, which has interesting applications, we give it here independently of other more general considerations.

2. Simplicial covering and harmonic chains. To compute the homology groups of $P$ and $\bar{P}$, let $K$ and $\bar{K}$ be finite simplicial complexes which are subdivisions of $P$ and $\bar{P}$ respectively, such that each oriented simplex of $K$ covers one oriented simplex of $\bar{K}$. Then $G$ acts as an automorphism group $p^{1}$ on $K$; the automorphisms $x \in G$ are permutations of the oriented simplices $\sigma_{n}$ of $K$ in each dimension $n$ and preserve all incidence relations. The set of all simplices $\sigma_{n}$ covering a simplex $\bar{\sigma}_{n}$ of $\bar{K}$ is a transitivity domain of $G$; that is, it contains with any simplex $\sigma_{n}^{\prime}$ all $x \sigma_{n}^{\prime}, x \in G$, and only those. Furthermore, since in an automorphism $x \neq e$ no simplex is fixed,

Presented to the Society, April 17, 1948; received by the editors February 25, 1948.

${ }^{1}$ For details see for example $[1, \S 6]$. Numbers in brackets refer to the references at the end of the paper. 
$x \sigma_{n}^{\prime}=x_{1} \sigma_{n}^{\prime}$ implies $x=x_{1}$. The simplicial map which projects each $\sigma_{n}$ of $K$ onto the $\bar{\sigma}_{n}$ it covers is denoted by $U$; obviously, $U x \sigma_{n}=U \sigma_{n}$ for all $x \in G$.

In the simplicial complexes $K$ and $\bar{K}$ we shall only use chains with real coefficients, and no distinction will be made between chains and cochains. An $n$-chain $a_{n}$ in $K$ is a linear form $a_{n}=\sum_{i} \alpha_{i} \sigma_{n i}$ with real coefficients, $\alpha_{i}$, where the $n$-simplices $\sigma_{n i}$ of $K$ play the role of indeterminates. All $n$-chains $a_{n}$ in $K$ form a vector space $C_{n}$ (a real linear space) of finite rank, in which the scalar product of $a_{n}=\sum_{i} \alpha_{i} \sigma_{n i}$ and $b_{n}=\sum_{i} \beta_{i} \sigma_{n i}$ is defined by $a_{n} \cdot b_{n}=\sum_{i} \alpha_{i} \beta_{i}$. The boundary operator $\partial$ is a linear mapping of $C_{n}$ into $C_{n-1}, n=0,1, \cdots\left(C_{-1}=0\right)$; the coboundary operator $\delta$ is the linear mapping of $C_{n-1}$ into $C_{n}$ which is $d u a l$ to $\partial$, that is, $\delta$ is defined by $\delta a_{n-1} \cdot b_{n}=a_{n-1} \cdot \partial b_{n}$ for all $(n-1)$ chains $a_{n-1}$ and $n$-chains $b_{n}$. If $\partial a_{n}=\delta a_{n}=0, a_{n}$ is called a harmonic chain; as in each homology or cohomology class there is exactly one harmonic chain, ${ }^{2}$ the vector group $H_{n}$ of all harmonic $n$-chains (a linear subgroup of $C_{n}$ ) is isomorphic both to the $n$th homology and the $n$th cohomology group of $K$ (the isomorphism is given by representing a homology or cohomology class by the harmonic chain it contains). The rank of $H_{n}$ is the $n$th Betti number $p_{n}$ of $K$. All chains, groups, and so on, of $\bar{K}$ will be denoted by the same symbols as in $K$, with a bar (for example, $\bar{a}_{n}, \bar{C}_{n}, \bar{H}_{n}$ ), the boundary and coboundary operator as in $K$ by $\partial$ and $\delta$.

The linear mappings of the chain groups and the homology groups induced by the simplicial maps $U$ and $x \in G$ will also be denoted by $U$ and $x$ respectively. $x$ is an isomorphism of $C_{n}$ onto itself, $U$ a homomorphism of $C_{n}$ onto $\bar{C}_{n}$, for all $n$.

The simplicial map $U$ of $K$ onto $\bar{K}$ is locally one-one; that is, if the simplices $\sigma_{n}$ and $\sigma_{n}^{\prime}$ of $K$ are both incident with a simplex $\sigma_{k}$, then $U \sigma_{n}=U \sigma_{n}^{\prime}$ implies $\sigma_{n}=\sigma_{n}^{\prime}$. For each simplex $\sigma_{n}$ of $K$ one has therefore not only $U \partial \sigma_{n}=\partial U \sigma_{n}$, but also

$$
U \delta \sigma_{n}=\delta U \sigma_{n}
$$

in other words, the linear mapping $U$ of $C_{n}$ onto $\bar{C}_{n}$ commutes with $\partial$ and $\delta$. Let $U^{*}$ be the dual mapping of $\bar{C}_{n}$ into $C_{n}$, defined by $U^{*} \bar{a}_{n} \cdot b_{n}$ $=\bar{a}_{n} \cdot U b_{n}$ for all $n$-chains $\bar{a}_{n} \in \bar{C}_{n}$ and $b_{n} \in C_{n}$; it also commutes with $\partial$ and $\delta$. Hence

(2) $U$ and $U^{*}$ both map harmonic chains onto harmonic chains.

Since $U$ is a mapping of $C_{n}$ onto $\bar{C}_{n}$, the dual mapping $U^{*}$ is an isomorphism of $\bar{C}_{n}$ into $C_{n}$. For, $U^{*} \bar{a}_{n}=0$ implies $U^{*} \bar{a}_{n} \cdot b_{n}=\bar{a}_{n} \cdot U b_{n}=0$

${ }^{2}$ See for example [2, pp. 245-246]. Other references are given in [2]. 
for all $b_{n} \in C_{n}$, hence $\bar{a}_{n} \cdot \bar{b}_{n}=0$ for all $\bar{b}_{n} \in \bar{C}_{n}$, hence $\bar{a}_{n}=0$. This together with (2) yields

TheOREM 2. $U^{*}$ induces an isomorphism of $\bar{H}_{n}$ into $H_{n}, n=0,1, \cdots$.

COROLLARY 1. The nth Betti number $\bar{p}_{n}$ of $\bar{K}$ is not greater than the nth Betti number $p_{n}$ of $K$.

We remark further that $U$ maps $U^{*} \bar{C}_{n} \subset C_{n}$ isomorphically onto $\bar{C}_{n}$. For, $U U^{*} \bar{a}_{n}=0$ implies $U U^{*} \bar{a}_{n} \cdot \bar{a}_{n}=U^{*} \bar{a}_{n} \cdot U^{*} \bar{a}_{n}=0$, hence $U^{*} \bar{a}_{n}$ $=0$. In particular $U$ maps $U^{*} \bar{H}_{n} \subset H_{n}$ isomorphically into $\bar{H}_{n}$, and since $U^{*} \bar{H}_{n}$ is isomorphic to $\bar{H}_{n}$, this must be a map onto $\bar{H}_{n}$. Hence

(3) $U$ maps $H_{n}$ onto $\bar{H}_{n}$.

REMARK, $U U^{*}$ is not the identity mapping of $\bar{C}_{n}$ or $\bar{H}_{n}$ but, as is easily seen, multiplies each chain $\bar{a}_{n}$ by the order $g$ of $G$.

3. Invariant chains. In the linear mapping of $C_{n}$ onto itself induced by an automorphism $x \in G$ the scalar product of two $n$-chains $a_{n}, b_{n}$ remains unchanged:

$$
x a_{n} \cdot x b_{n}=a_{n} \cdot b_{n} .
$$

This follows simply from the fact that $x$ is a permutation of the oriented simplices (that is, of the basis vectors of $C_{n}$ ). In other words, $x$ is an orthogonal transformation of $C_{n}$.

A chain $a_{n} \in C_{n}$ will be called invariant if $x a_{n}=a_{n}$ for all $x \in G$. This is the case if and only if

$$
a_{n} \cdot b_{n}=a_{n} \cdot x b_{n}
$$

for all $x \in G$ and $b_{n} \in C_{n}$. For, if $a_{n}$ is invariant, $a_{n} \cdot b_{n}=x a_{n} \cdot x b_{n}$ $=a_{n} \cdot x b_{n}$; and conversely, if (5) holds for all $x \in G$, then $a_{n} \cdot b_{n}=a_{n}$ $\cdot x^{-1} b_{n}=x a_{n} \cdot b_{n}$ for all $b_{n} \in C_{n}$, hence $a_{n}=x a_{n}$. The invariant chains constitute a linear subgroup $C_{n}^{i}$ of $C_{n}$.

(6) The isomorphism $U^{*}$ maps $\bar{C}_{n}$ onto $C_{n}^{b}$.

Proof. For any $\bar{a}_{n} \in \bar{C}_{n}, U^{*} \bar{a}_{n}$ is invariant; for

$$
U^{*} \bar{a}_{n} \cdot x b_{n}=\bar{a}_{n} \cdot U x b_{n}=\bar{a}_{n} \cdot U b_{n}=U^{*} \bar{a}_{n} \cdot b_{n}
$$

for all $b_{n} \in C_{n}$. Conversely, if $a_{n}$ is invariant, the relation

$$
\bar{a}_{n} \cdot U \sigma_{n}=a_{n} \cdot \sigma_{n} \quad \text { for all } \sigma_{n} \text { of } K
$$

defines an $n$-chain $\bar{a}_{n}$ of $\bar{K}$ without ambiguity (for replacing $\sigma_{n}$ by $\sigma_{n}^{\prime}=x \sigma_{n}$ does not change either side of this equation). Then $U^{*} \bar{a}_{n} \cdot \sigma_{n}$ $=\bar{a}_{n} \cdot U \sigma_{n}=a_{n} \cdot \sigma_{n}$ for all $\sigma_{n}$, hence $U^{*} \bar{a}_{n}=a_{n}$.

The invariant harmonic $n$-chains of $K$ form a linear subgroup $H_{n}^{t}$ of $H_{n}$. For any $a_{n} \in H_{n}^{i}$ there is, by (6), an $\bar{a}_{n}$ such that $a_{n}=U^{*} \bar{a}_{n}$; 
since $U^{*}$ commutes with $\partial$ and $\delta$ and is an isomorphism, it follows that $\bar{a}_{n}$ is harmonic. Combining this with Theorem 2 and (6) we obtain the following result.

TheOREM 3. $U^{*}$ induces an isomorphism of $\bar{H}_{n}$ onto $H_{n}^{t}$.

4. Proof of Theorem 1. Since the automorphisms $x \in G$ of $K$ permute the $n$-simplices, for each $n$, and preserve the incidence relations, the linear mappings $x$ of $C_{n}$ onto itself, $n=0,1, \cdots$, commute with both $\partial$ and $\delta$. Hence they induce linear mappings of $H_{n}$ onto itself; these mappings describe the operation of $G$ on the $n$-dimensional homology and cohomology groups of $K$. By (4) these linear mappings of $H_{n}$ onto itself are orthogonal. Hence for each $n$ one has an orthogonal representation $\mathcal{R}_{n}$ of the group $G$ in the vector group $H_{n}$. The subspace $H_{n}^{i}$ of $H_{n}$ consists of those elements of $H_{n}$ which are fixed under this representation; by Theorem 3 it may be considered as the homology or cohomology group $\bar{H}_{n}$ of $\bar{K}$. This proves that the latter is determined by $H_{n}$ and by the operation of $G$ in $H_{n}$ (cf. $\$ 1$ ).

The rank of $H_{n}^{t}$, which is the $n$th Betti number $\bar{p}_{n}$ of $\bar{K}$, is equal to the number of trivial irreducible representations of $G$ contained in the representation $R_{n}$ of $G$ in $H_{n}$, hence by a well known character relation $^{3}$ is equal to the average over the group $G$ of the character $s_{n}(x)$ of the representation $R_{n}$. This proves Theorem 1. Replacing $s_{n}(e)$ by the degree $p_{n}$ of the representation, where $e$ is the unit element of $G$, we may write the formula in the form

$$
g \cdot p_{n}=p_{n}+\sum_{x \in G, x \neq e} s_{n}(x) .
$$

REMARK. $p_{n}=p_{n}$ holds if and only if $H_{n}=H_{n}^{b}$, hence if all harmonic $n$-chains of $K$ are invariant under the operations of $G$; in other words, if each $n$-dimensional homology class is mapped into itself by all $x \in G$. This result may be stated in the following way:

THEOREM 4. The nth Betti numbers $p_{n}$ and $p_{n}$ of $K$ and $\bar{K}$ are equal if and only if in the dimension $n$ all automorphisms $x \in G$ are of the homology type of the identity (for real coefficients). ${ }^{4}$

5. Products, ${ }^{5}$ manifolds. The Alexander $\cup$-product associates with two cohomology classes of $K$ of dimensions $n$ and $k$ a third one of di-

${ }^{3}$ Cf. [3, p. 201, formula (16)].

4 The sufficiency of the condition has previously been proved by G. Hirsch, with an application to closed Lie groups; see [4, p. 226].

- For definitions and properties see [5]; for products in a covering complex the remarks of $[1, \S 6]$ have to be used. 
mension $n+k$, hence with two harmonic chains $a_{n}$ and $b_{k}$ a unique harmonic $(n+k)$-chain $a_{n} \cup b_{k}$. The Čech-Whitney $\cap$-product associates with a cohomology class of dimension $k$ and a homology class of dimension $k+p(p \geqq 0)$ a homology class of dimension $p$, hence with two harmonic chains $b_{k}$ and $c_{k+p}$ a unique harmonic $p$-chain $b_{k} \cap c_{k+p}$. From the fact that all incidence relations are preserved by the automorphisms $x \in G$ it may easily be deduced that

$$
\begin{aligned}
x a_{n} \cup x b_{k} & =x\left(a_{n} \cup b_{k}\right), \\
x b_{k} \cap x c_{k+p} & =x\left(b_{k} \cap c_{k+p}\right)
\end{aligned}
$$

for all harmonic chains $a_{n}, b_{k}, c_{k+p}$ and all $x \in G$.

It follows that the $U$ - or $\cap$-product of invariant harmonic chains is again an invariant harmonic chain. The direct sum of all $H_{n}^{b}$, $n=0,1, \cdots$, together with the $U$-product, constitutes a subring $R^{i}$ of the Alexander ring (the cohomology ring) $R$ of $K$. Since $U^{*}$ preserves the $U$-product, it follows from Theorem 3:

(10) $U^{*}$ induces a ring-isomorphism of the cohomology ring $\bar{R}$ of $\bar{K}$ onto $R^{i}$.

We now assume $K$ to be a (closed) orientable $N$-dimensional manifold. Let $m_{N}$ be the fundamental harmonic $N$-chain corresponding to an orientation of $K$; that is, $m_{N}$ is a chain with coefficients \pm 1 generating $H_{N}$. The duality operator $D$ in $K$ may be defined by

$$
D a_{n}=a_{n} \cap m_{N}
$$

for harmonic chains $a_{n}$; if $a_{n}$ is considered as a representative of an $n$-dimensional cohomology class, $D a_{n}$ is the harmonic representative of the dual $(N-n)$-dimensional homology class. As is well known, $D$ is an isomorphism of $H_{n}$ onto $H_{N-n}$.

(12) For any $x \in G$ one has $x D a_{n}=\gamma \cdot D x a_{n}$, where $\gamma= \pm 1$ is the (topological) degree of $x$.

PROOF. $x D a_{n}=x\left(a_{n} \cap m_{N}\right)=x a_{n} \cap x m_{N}=x a_{n} \cap \gamma m_{N}=\gamma \cdot\left(x a_{n} \cap m_{N}\right)$ $=\gamma \cdot D x a_{n}$.

As a consequence we have:

Theorem 5. If $K$ is an orientable $N$-dimensional manifold, then the characters $s_{n}(x)$ and $s_{N-n}(x)$ of the representations of $G$ in $H_{n}$ and $H_{N-n}$ are related by

$$
s_{n}(x)=\gamma \cdot s_{N-n}(x),
$$

where $\gamma$ is the degree of $x \in G$.

6. An application. Let $\bar{K}$ be a non-orientable $N$-dimensional manifold, and $K$ an orientable two-sheeted covering of $K$. The cover- 
ing transformation group consists of the unit element $e$ and an element $y$ of degree -1 . Various relations between the Betti numbers of $\bar{K}$ and $K$ may be deduced from (7) and (12). (7) becomes in this case (we write $s_{n}$ for $s_{n}(y)$ )

$$
2 p_{n}=p_{n}+s_{n}, \quad n=0,1, \cdots, N .
$$

Since $y$ has degree -1 , one has, by (12), $s_{n}=-s_{N-n}$, hence $2 p_{N-n}$ $=p_{N-n}+s_{N-n}=p_{n}-s_{n}=2 p_{n}-2 s_{n}$, hence

$$
\begin{aligned}
& p_{n}-p_{N-n}=s_{n}, \\
& p_{n}+p_{N-n}=p_{n} .
\end{aligned}
$$

This is a duality theorem for non-orientable $N$-dimensional manifolds. We shall use (13)-(15) to prove the following theorem.

THEOREM 6. The following relations hold between the Betti numbers $p_{n}$ of a closed non-orientable $N$-dimensional manifold $\bar{K}$ and the Betti numbers $p_{n}$ of a two-sheeted orientable covering $K$ of $\bar{K}$ :

(16) for $N=3, \quad p_{1}=2 p_{1}-1 ;^{6}$

(17) for $N=2 L, \quad p_{L}=2 p_{L}$;

$$
\text { for } \begin{gathered}
N=2 L+1, \sum_{n=0}^{L}(-1)^{n} p_{n}=2 \sum_{n=0}^{L}(-1)^{n} \bar{p}_{n} \quad \text { and } \\
\sum_{n \text { even }} p_{n}=2 \sum_{n \text { even }} p_{n} .
\end{gathered}
$$

Proof. (a) If $N=3$, the Euler characteristic $p_{0}-p_{1}+p_{2}-p_{3}=0$, where $p_{0}=1, p_{3}=0$, hence $p_{2}=\bar{p}_{1}-1$; by $(15), p_{1}+p_{2}=2 p_{1}-1=p_{1}$. (b) (17) follows immediately from (15). (c) If $N=2 L+1$, the characteristics of $K$ and $\bar{K}$ are 0 and we deduce from (13)

$$
\sum_{n=0}^{N}(-1)^{n} s_{n}=0
$$

hence $\sum_{n=0}^{L}(-1)^{n} s_{n}=-\sum_{n=L+1}^{N}(-1)^{n} s_{n}$; since $s_{n}=-s_{N-n}$, this is equal to $-\sum_{n=L+1}^{N}(-1)^{N-n} s_{N-n}=-\sum_{n=0}^{L}(-1)^{n} s_{n}$, hence $\sum_{n=0}^{L}(-1)^{n} s_{n}=0$. Using $s_{n}=-s_{N-n}$ again, we may also write this as $\sum_{n \text { even }} s_{n}=0$. These two results together with (13) yield (18). We remark that since the characteristics of $K$ and $\bar{K}$ are $0(N=2 L+1)$ the first of the formulas (18) is equivalent to $\sum_{n=L+1}^{N}(-1) p_{n}$ $=2 \sum_{n=L+1}^{N}(-1) p_{n}$, the second one to $\sum_{n \text { odd }} p_{n}=2 \sum_{n \text { odd }} \bar{p}_{n}$.

- For 3-dimensional manifolds $\bar{K}$ satisfying certain restrictive conditions (16) is proved in a note by T. H. Kiang [6]. 
REMARK. The formula (19), which here was proved directly, may also be deduced from a well known result concerning the number of fixed simplices in a simplicial self-mapping. ${ }^{7}$ In the general case of an arbitrary automorphism group $G$ and arbitrary finite complexes $K$ and $\bar{K}$ it follows from this result that the "Lefschetz number" $\lambda(x)=\sum_{n=0}^{N}(-1)^{n} s_{n}(x)$ is 0 for any automorphism $x \neq e$.

\section{REFERENCES}

1. B. Eckmann, Der Cohomologiering einer beliebigen Gruppe, Comm. Math. Helv. vol. 18 (1945-1946) pp. 232-282.

2. - Harmonische Funktionen und Randwertaufgaben in einem Komplex, Comm. Math. Helv. vol. 17 (1945) pp. 240-255.

3. B. L. van der Waerden, Moderne Algebra, vol. 2, Berlin, 1931.

4. G. Hirsch, Sur les groupes d'homologie de certains complexes de recouvrement, Portugaliae Math. vol. 4 (1945) pp. 225-237.

5. H. Whitney, On products in a complex, Ann. of Math. vol. 39 (1938) pp. 397432.

6. T. H. Kiang, An application of the addition formulas of Mayer-Vietoris, Academia Sinica Science Record vol. 7 (1945) pp. 275-276.

7. P. Alexandroff and H. Hopf, Topologie, Berlin, 1935.

INSTITUTE FOR AdVANCED STUdy aNd

UNIVERSITY OF LAUSANNE

${ }^{7}$ Cf. $[7$, p. 530]. 